\title{
VHP: Vibrotactile Haptics Platform for On-body Applications
}

\author{
Artem Dementyev \\ Google Research \\ Mountain View, CA, USA \\ artemd@google.com
}

\author{
Pascal Getreuer \\ Google Research \\ Mountain View, CA, USA \\ getreuer@google.com
}

\author{
Dimitri Kanevsky \\ Google Research \\ Mountain View, CA, USA \\ dkanevsky@google.com
}

\author{
Malcolm Slaney \\ Google Research \\ Mountain View, CA, USA \\ malcolmslaney@google.com
}

\author{
Richard Lyon \\ Google Research \\ Mountain View, CA, USA \\ dicklyon@google.com
}

\begin{abstract}
Wearable vibrotactile devices have many potential applications, including sensory substitution for accessibility and notifications. Currently, vibrotactile experimentation is done using large lab setups. However, most practical applications require standalone on-body devices and integration into small form factors. Such integration is time-consuming and requires expertise.

With a goal to democratize wearable haptics we introduce VHP, a vibrotactile haptics platform. It includes a low-power miniature electronics board that can drive up to 12 independent channels of haptic signals with arbitrary waveforms at a $2 \mathrm{kHz}$ sampling rate. The platform can drive vibrotactile actuators including linear resonant actuators and voice coils. The control hardware is battery-powered and programmable, and has multiple input options, including serial and Bluetooth, as well as the ability to synthesize haptic signals internally. We developed current-based loading sensing, thus allowing for unique features such as actuator auto-classification, and skin-contact quality sensing. Our technical evaluations showed that the system met all our initial design criteria and is an improvement over prior methods as it allows all-day wear, has low latency, has battery life between 3 and 25 hours, and can run 12 actuators simultaneously.

We demonstrate unique applications that would be timeconsuming to develop without the VHP platform. We show that VHP can be used as bracelet, sleeve and phone-case form factors. The bracelet was programmed with an audio-to-tactile interface and was successfully worn for multiple days over months by developers. To facilitate more use of this platform, we open-source our design and plan to make the hardware widely available. We hope this work will motivate the use and study of vibrotactile all-day wearable devices.
\end{abstract}

\section{CCS CONCEPTS}

- Human-centered computing $\rightarrow$ Haptic devices.

CC)
This work is licensed under a Creative Commons Attribution International
4.0 License.
UIST '21, October $10-14,2021$, Virtual Event, USA
(C) 2021 Copyright held by the owner/author(s).
ACM ISBN 978-1-4503-8635-7/21/10.
https://doi.org/10.1145/3472749.3474772

\section{KEYWORDS}

haptics, vibrotactile, accesibility, platforms, wearable

\section{ACM Reference Format:}

Artem Dementyev, Pascal Getreuer, Dimitri Kanevsky, Malcolm Slaney, and Richard Lyon. 2021. VHP: Vibrotactile Haptics Platform for On-body Applications. In The 34th Annual ACM Symposium on User Interface Software and Technology (UIST '21), October 10-14, 2021, Virtual Event, USA. ACM, New York, NY, USA, 15 pages. https://doi.org/10.1145/3472749.3474772

\section{INTRODUCTION}

Vibrotactile actuators are typically electric vibration motors designed to elicit tactile sensation. Nearly all wearable devices and mobile phones employ vibrotactile actuators, as the advantages of haptics in small devices have been well documented [40]. Haptic usage is accelerating due to advances in technology, such as improvements in linear resonant actuators (LRA) and electronics miniaturization.

Haptics research requires flexible multi-channel actuators. For example, reproducing a popular haptics experiment such as cutaneous rabbit illusion [16] requires at least three actuators that can produce specific waveforms. Previous research showed that multiple vibrotactile actuators increase the information transfer rate in haptic-based communications [50]. Today's options require bulky and expensive multi-channel audio amplifiers, customized software, and messy wiring. This can be seen in our initial setup in Figure 1B. Such devices are not wearable in everyday life. On the other hand, highly integrated haptic actuators such as ones inside our mobile phones provide small form factors but do not allow any customization, such as using different actuators or locations. Also, they are usually limited to one or two actuators. Furthermore, closed systems do not allow software and hardware changes, which could be significant in accessibility use, where each user's needs are unique.

In compelling examples, vibrotactile hats, belts, watches, and headbands have been suggested as a suitable channel of communication for accessibility, for instance, for hard-of-hearing [18] and visual impairments $[13,30]$. Also, vibrotactile stimulation can reduce the need for visual attention for alert perception. One study showed that 24 alert patterns could be distinguished on the wrist [27]. However, to effectively field-test such technology outside the laboratory requires experimentation with multi-channel on-body devices in miniaturized form factors. The barrier to developing specialized miniaturized hardware and software is high. 

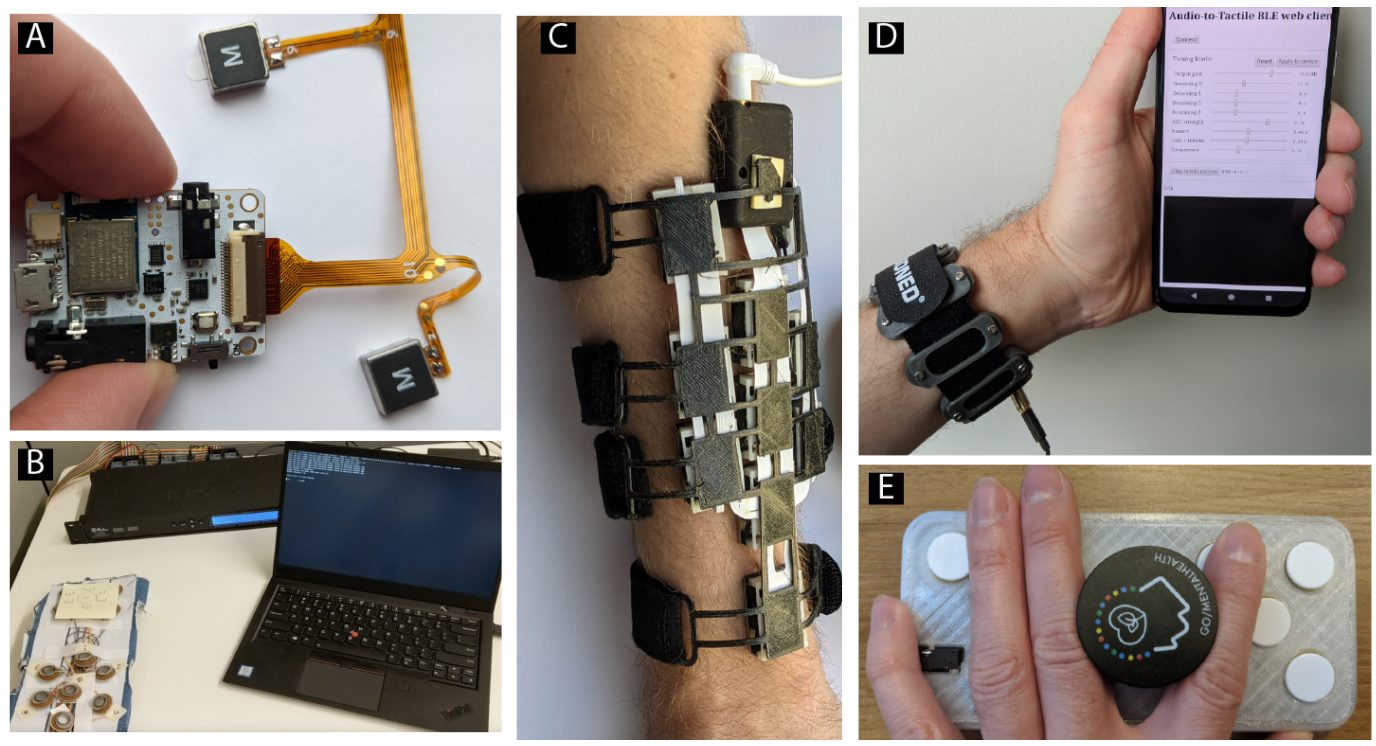

Figure 1: A) The main component of the Vibrotactile Haptics Platform (VHP) is the 12-channel output programmable driver board. The board is connected with a flexible PCB to multiple linear resonant actuators (two shown here). B) In comparison, the typical lab setup requires a large audio interface (developed by MOTU, behind the computer), audio amplifiers in addition to a computer. C) A sleeve with the VHP and ten actuators. D) An 8-actuator bracelet powered by the VHP. An on-board battery powers the bracelet. We developed an audio-to-tactile application on this device and a Bluetooth interface, as shown on a mobile phone. E) A phone case with eight tactors, driven by the VHP.

As there is currently nothing in between lab setups and highly integrated consumer products, taking haptic experiments beyond the lab setting and into daily wearable devices requires a significant amount of time and expertise in electronics and software. One would have to design a small custom circuit board with amplifiers and signal generators complicated by the high power requirements of haptic actuators and demanding data bandwidth for multi-channel playback. Furthermore, haptics should be optimized for low latency; thus, an experimenter needs to write highly optimized firmware to meet this requirement.

Motivated by our desire to experiment with wearable multichannel haptics, we developed a platform that we believe is useful for others as well. In this paper, we present a haptics platform that provides a 12-channel haptic driver in a highly integrated form factor, as shown in Figure 1. The device can be battery-powered, and integrated into wearable devices and small gadgets, while supporting high-bandwidth haptic communications. Figure 1 shows some devices developed on our platform. The haptic actuators can be controlled by signals arriving via serial, USB, Bluetooth Low Energy (BLE), as well as onboard microphones, using an onboard programmable processor. The contact area, type of tissue, and pressure can load and significantly change the behavior of the actuator [4], thus affect perception. In our design, we measure the current driving each vibrotactile actuator, allowing us to sense its loading. To facilitate widespread use of the platform, we open-source our software and hardware design and plan to make manufactured hardware available from a distributor.
This paper is structured as follows. First, we describe previous work to position this platform in relation to existing haptic toolkits, devices and actuators. Then we discuss the design principles that guided the development of the platform, informed by previous work and our own experience. Later we present and explain the design of the hardware and the software. We follow this with a technical evaluation of the essential system parameters. To demonstrate how this platform can be used in real-life, we develop bracelet, sleeve and phone case form factors, and use them in practical applications. Finally, we describe limitations, future work, and give concluding remarks.

\subsection{Contributions}

- Open-sourced hardware and software platform for wearable haptics. The hardware is composed of a custom-designed miniaturized circuit board that can drive up to 12 haptic channels. The firmware is built on an Arduino-compatible platform and allows multiple input/output options, as well as local processing and signal generation. The platform provides an improvement over prior solutions, as it allows all day wear. The code and design files are available via GitHub [19].

- Ability to sense the actuator loading. To enable sensing, we integrated custom high-speed current sensing for every channel. This feature enables unique abilities of our platform, such as skin contact sensing and actuator auto-identification.

- Applications and use cases of the platform. We use this platform to quickly iterate integrated wearable devices such as 
a bracelet, phone case, and sleeve. We describe the experience of wearing the bracelet as an all-day audio-to-tactile substitution, and the use of the platform in psychophysical tactile experiments. We show how the ability to sense the loading can be applied to a phone case to turn off actuators that are not in contact with fingers.

- Technical characterization including power consumption, bandwidth, latency, and sensing the loading.

\section{PREVIOUS WORK}

This section reviews related work. First, we look at existing toolkits for haptics. Then we examine existing wearable vibrotactile devices in the commercial and research spaces. Finally, we look at selfsensing on vibrotactile actuators.

\subsection{Vibrotactile Haptics Toolkits}

Researchers have developed vibrotactile and non-vibrotactile haptic toolkits and platforms from which we draw inspiration for this work. Also, there are a small number of commercial toolkits, mainly targeting mobile phone haptics.

In the space of vibrotactile-based haptics toolkits, one of the closest research projects to ours is Syntacts platform [39]. This project developed an 8-channel custom amplifier driven by an audio interface and a custom graphical user interface to generate waveforms. The resulting device is bulky, as for 12-channels it needs a $1 \mathrm{~kg}$ and $22 \mathrm{~cm}$ long amplifier (UltraLiteMk4, MOTU), 12 audio cables, and a connection to a PC. Such a setup is acceptable for a lab experiment, but could not be used in a wearable scenario. The VibroPixels project [20] demonstrated scalable haptic devices that are controlled wirelessly and are battery-powered. A number of such devices can be strapped to the user. However, the devices are not designed for integration into daily wearable devices and are targeted towards artistic applications. Other vibrotactile-based haptic toolkits use the audio output of a PC, such as TECHTILE [32], Stereohaptics [23], Vitaki [31], TactiPEd [35], Music haptics [7]. However, none of these toolkits are suitable for wearable applications, and they all have a limited number of channels, and require a cable tethered to a PC. While driving audio has some of the same requirements as haptics experiments, haptics often require more channels and may use a lower sample rate and bit depth than what is typically used for audio signals. Therefore, we designed our device without reliance on audio solutions.

There is a diverse set of non-vibrotactile haptics toolkits. WoodenHaptics [14] provides a spatial haptics toolkit using wood components and force-feedback motors. Multiple inflatable soft materials platforms were utilized for haptic applications, especially in the tangible interfaces field [33, 34]. Other researchers explored electrotactile [26, 44], airflow [25] and temperature changes [37] to stimulate the skin. Currently, vibrotactile-based haptics are the most practical for wearable devices as they are compact, low power, simple to control, and easily available.

\subsection{Commercial Haptics Toolkits}

There are a number of hardware and software commercial solutions for delivering haptic signals. Android and iOS both provide application programming interfaces (API) $[2,10]$ to generate haptic waveforms on compatible devices. Furthermore, there are proprietary software tools, such as Lofelt Studio [29], used to create haptic effects for mobile phones.

There are specially designed driver chips, such as DRV2608 (Texas Instruments) [22], that have built-in effects libraries. They can be obtained in a breakout version [47] and have Arduino libraries for faster prototyping. However, such drivers are usually single-channel and require significant time investment for hardware and software to integrate into a wearable system.

To the best of our knowledge, none of the state-of-the-art commercial and research haptics toolkits can provide a flexible wearable and self-contained solution.

\subsection{Wearable Haptic Devices}

There are many wearable vibrotactile devices in the research and commercial worlds, albeit limited in several ways. Almost all modern mobile phones and smartwatches provide vibrotactile feedback. The devices come in various form factors and with different use cases. The devices are too numerous to list here, so we focus on accessibility examples, as we later apply our system in this area.

Several commercial devices provide audio-to-tactile conversion for users with hearing loss. One of the early examples is Kanievsky Sound [8] that provides two vibrotactile channels that are strapped on the body, usually around the wrist. The Tactaid series of devices [15] had between two to seven haptic channels that converted sound to vibrations. Unlike the older devices, which required a wired box with a battery and electronics, Buzz [38] is an all integrated wrist-worn audio-to-tactile device with four vibrotactile actuators. There have also been devices designed to provide haptic feedback during gaming, such as a vest [5] designed with 40 eccentric rotating motors. SUBPAC [48] is a large wearable speaker, in the form factor of a backpack that provides tactile bass. These commercial devices provide a high level of integration but do not allow for customization.

In the research literature, there are a large number of diverse vibrotactile devices, including, for example, a 24 vibrotactile actuator sleeve for speech communication [42]. BuzzWear (no relation to commercial Buzz watch) is a three vibrotactile actuator wrist-worn device for alert perception [27]. Wrist-worn MosaicOne [12] has four tactile actuators for audio-to-tactile conversion. Researchers also integrated vibrotactile actuators in a chair [49] and vest [55]. For visual-based assistive navigation, actuators were integrated into a belt [11] and a helmet [30].

Previous work demonstrates the diversity of possible vibrotactile applications. In all of those cases, a custom design had to be implemented to fit the various form factors, and could potentially be more quickly prototyped with our platform. Also, those devices could benefit from state-of-the-art features of our platform such as small form factor, wireless communications, long battery life, and sensing actuator loading to alert when the actuator is broken or not making proper contact with the skin.

\subsection{Self-Sensing Vibrotactile Actuators}

State-of-the-art commercial vibrotactile actuator drivers such as DRV2608 (Texas Instruments) employ back-electromotive-force 
(back-EMF) sensing to track peak resonance. Back-EMF is a selfinduced voltage due to the movement of the coils inside the actuator. Researchers have explored back-EMF for load sensing [3] and other uses such as touch and pressure sensing [9]. Other measurement techniques use accelerometers [17,21] or microphones [52] to measure the load on the vibrotactile actuators, but have not been used in practice.

The disadvantage of back-EMF is that it requires disconnecting the actuator to measure back-EMF reliably, thus needing a significant number of switches and a multiplexer with a large number of channels. While back-EMF would work for our platform, we use current sensing to minimize the board footprint and number of components. An electric motor consumes different amounts of current based on how fast it is moving and how much force it needs to generate. Current sensing is mainly used for torque control without the need for a mechanical sensor. For example, higher-end servo motors (e.g., Dynamixel [43]) often have this ability. However, to our knowledge, current sensing has not yet been applied to vibrotactile loading estimation.

To the best of our knowledge, automatic identification of the haptic actuator model has not been done before. However, artificial neural networks have been used to learn and identify control parameters of an electric motor by measuring known excitation signals (e.g., [54]). Such an approach can provide some plug-and-play ability to motors without manual tuning.

\section{DESIGN PRINCIPLES}

Based on previous work and our own experience, we developed the VHP design according to the following principles for the "ideal" wearable haptics platform.

Imagine a scenario where a user wants to make a vibrotactile device with six actuators for recognizing specific sounds by providing vibration alerts. We envision the user as someone with at least basic electronics and programming skills, such as a designer, engineer, or researcher. Initially, the user does not know the exact form factor. First, using our platform, one can quickly get started with a lab-bench prototype for testing multiple vibrotactile actuators and components with a breakout board. Further, one can use PC audio-editing software to create multichannel waveforms and drive the device with them through the USB interface. Such an approach has worked well in workshops, even with novice users [23, 32]. Once the parts and other aspects are decided, the user can implement a wearable prototype without modifying the electronics and software. All effort could be spent developing the signal processing and exploring form factors and wear tests using prototyping tools such as $3 \mathrm{D}$ printers. The user can wear the device all day to understand how it functions in practice. The device could be conveniently controlled with a mobile phone.

We believe the following design aspects are most important:

(1) All-day wrist-worn wearable capability. The platform should be comfortable to wear and able to function independently, without a tether to a PC or amplifier. Size should be limited to a common wristwatch, which is typically in the range of 38 to $46 \mathrm{~mm}$ in diameter. Such size is familiar to most users, and can integrate into most wearable form factors. Power consumption should be low enough to allow all-day wear on a small battery.

(2) Flexible connectivity with external devices. As the potential use cases of the platform are diverse, the tactile waveforms can be generated onboard or received from an external controller. The platform should have wired interface options, including serial and USB, and wireless such as Bluetooth. The wired connectivity is useful in streaming and generating haptic data when tethered to a PC, as shown by toolkits by other researchers [23, 32]. Wireless connectivity allows to control and monitor the system from a mobile phone during daily wear. This feature has been deployed in commercial devices such as Buzz [38].

(3) Optimized for vibrotactile-based haptics. The sample rate should be around $2 \mathrm{kHz}$, as tactile perception can be perceived from 1 to $1000 \mathrm{~Hz}$ with maximum sensitivity between 150 and $300 \mathrm{~Hz}$ [24]. The latency should be under $20 \mathrm{~ms}$ so that it is not noticeable [53]. In particular, it is desirable that tactile cues be perceptually synchronized with vision when using tactile signals to convey a representation of live audio (e.g., for lipreading). The number of channels should be large to allow driving multiple actuators simultaneously. The number of channels used in research varies from 1 to 24 [51]. We limit the design to electric motor-based vibrotactile haptics (e.g., voice coils, LRAs, eccentric rotating motors (ERM)), as they are compact, widely available, and simple to drive.

(4) Loading-sensing actuators. For consistent control over stimulation, vibrotactile actuators require proper contact with the skin. The device should measure skin contact and provide an alert or self-adjust if it is not loaded correctly. The system should also provide self-testing to help debug the wiring of the actuators.

(5) Available hardware. Even with access to open-sourced designs, manufacturing hardware can be an intimidating process that excludes many potential users. Thus, we believe it is essential to provide hardware tests, manufacturing and fulfillment in addition to open-sourced design. Access to manufactured hardware has contributed to the wide adaptation of research projects such as CircuitStickers [41].

(6) Open software and firmware. We believe firmware should be written using widely accessible platforms platform (e.g., Arduino) and languages such as Python to allow users to quickly create and share the code and leverage open-source drivers (e.g., bootloader, USB). Thus, to fully use and customize the VHP platform, the user should have some programming and electronics familiarity.

\section{HARDWARE DESIGN}

Figure 2 shows the system diagram. We created a custom 6-layer printed circuit board (PCB) as shown in Figure 3. We used the nRF52840 (Nordic Semiconductor) as the main processor. This microcontroller offers a high degree of integration. The processor has sizeable computer power, offering a $64 \mathrm{MHz}$ Cortex M4 processor with a floating-point, and it is capable of driving up to 12 independent channels with arbitrary waveforms with pulse-width 


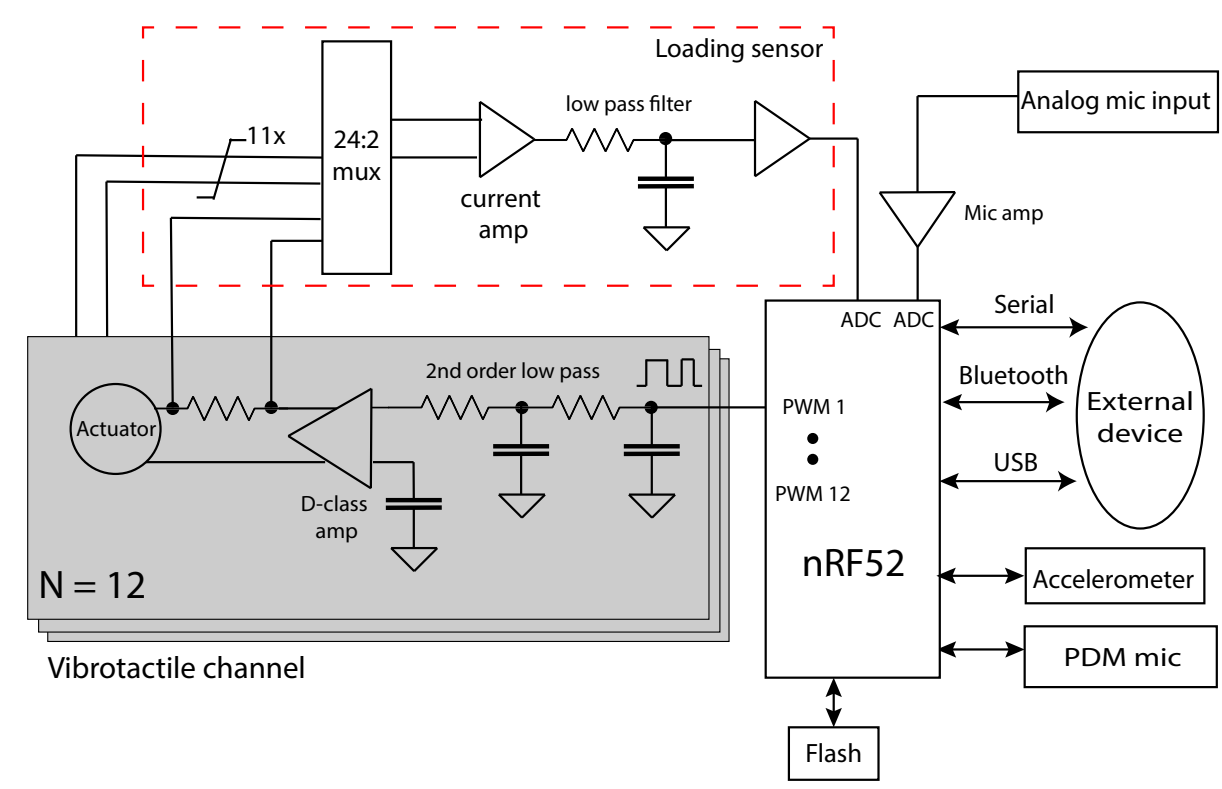

Figure 2: Block diagram of the system design, showing the main components. The external device includes a PC, mobile phone, or an embedded microcontroller. To save space, only one of the twelve vibrotactile channels is shown. The loading sensing channels are multiplexed from 24 to 2 using pair of 16:2 analog multiplexors.

modulated (PWM) output signals. We employ Bluetooth, USB, and serial for communications with external devices. We adapted a 4conductor $3.5 \mathrm{~mm}$ audio jack to carry external power and serial communications. The audio cable is easily available, and is usually compact and comfortable for wearable applications. We also added a 16 MBit flash storage (GD25Q16, ELM Technology).

\subsection{Power}

The main design challenge is the need to provide high current for the actuators in a small package. The actuators' current draw can be spiky, and the total peak current can be as high as $5.5 \mathrm{~A}$, if all actuators are on. We increased the design's robustness to current spikes by having multiple ground and power planes, and adding multiple large capacitors.

The electronics were designed to reduce power consumption, where every component can be independently shut down using a switch or a shut-down pin.

The board can be powered by a lithium-polymer battery with a small 2-pin JST SH connector or externally through a $3.5 \mathrm{~mm}$ TRRS audio jack. The board also contains a lithium-polymer charger, which can be used to charge the battery over a Micro USB port.

\subsection{Connecting Haptic Actuators}

The interface between the board and actuators is critical, as the 12 tactile signals (24 total wires) have to be quick to set up, robust for wearable application, and flexible. For the interface, we use a 24-pin FPC (flexible printed circuit) connector on the VHP. We support interfacing to the actuators in two ways:

(1) Using a custom flexible circuit board. Some examples of custom boards are shown in Figure 4. This option provides the smallest size, best aesthetics, robustness, and fastest assembly, at the expense of less design flexibility and dedicated design and manufacturing time. Often times in a wearable device meant to be worn for an extended time, this is the best option. In this situation, the flexible circuit is a simple one or two-layer, with no parts on it. Such boards can be manufactured inexpensively in one or two weeks from $\$ 10$ to $\$ 20$ per board by many manufacturers. (e.g. PCBWay ${ }^{1}$, OSHPark $^{2}$ )

(2) Using a rigid breakout board. This option provides the ability to reconfigure the design quickly. It is best used for lab setups and early prototypes. The breakout board is connected to the haptic board through an off-the-shelf ribbon FPC cable (e.g. 0150210624, Molex) or a custom FPC cable, as shown in Figure 4A. The breakout board contains a screw terminal to quickly connect and disconnect actuators and pins for breadboard-friendly jumper cables. However, many modern small actuators don't have wire leads, but only pads. In such cases, wires would need to be soldered to the actuators' pads for interfacing.

\subsection{Vibrotactile Driver Design}

We use all three of the microcontroller's PWM modules to generate 12 independent PWM signals, as each module creates four signals. The signals are generated at $15.625 \mathrm{kHz}$. To avoid having the actuator produce an audible whine, the PWM carrier is suppressed with a passive second-order analog low pass filter with a $450 \mathrm{~Hz} 3 \mathrm{~dB}$ corner frequency. Then the waveforms are passed to

\footnotetext{
${ }^{1}$ https://www.pcbway.com/

${ }^{2}$ https://oshpark.com/
} 


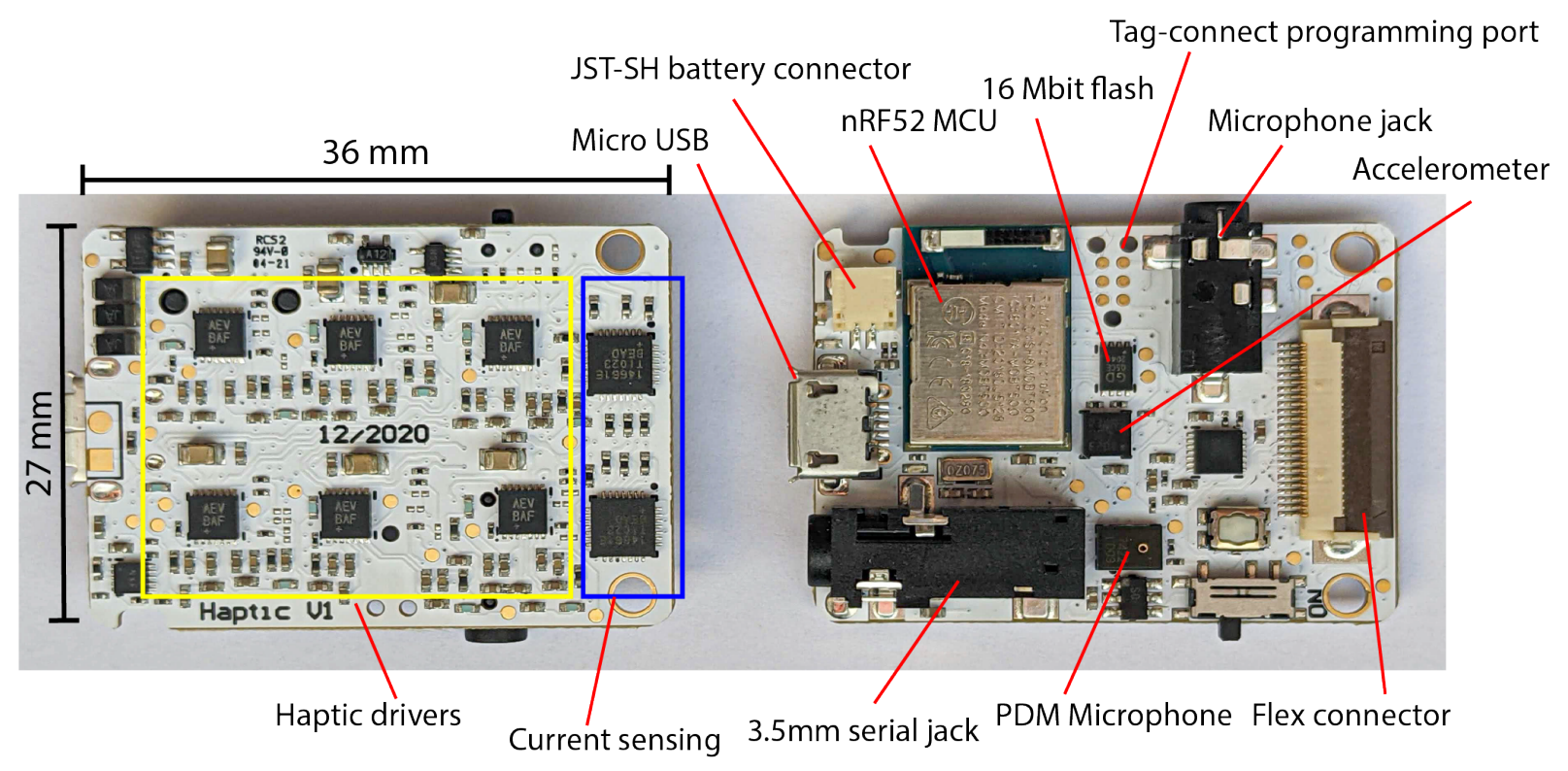

Figure 3: Front and back sides of the main 6-layer circuit board. Key components are labeled.
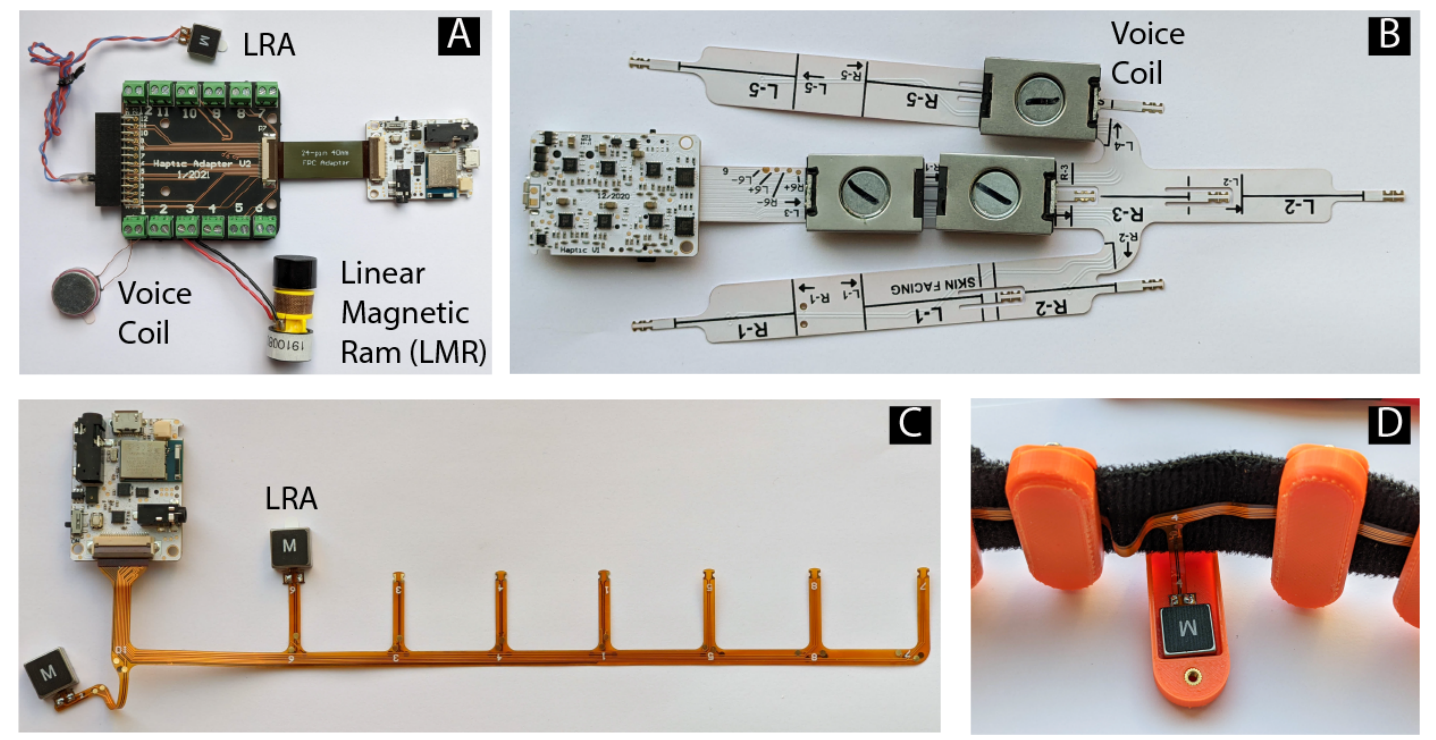

Figure 4: Different ways to connect multiple vibrotactile actuators. A) Using a breakout board with screw terminal connectors attached to three different vibrotactile actuators. Wire leads were soldered to the LRA, as it only has pads for attachment. B) Using a custom flex PCB for a forearm sleeve. Three of the ten vibrotactile actuators are attached. C) Custom flex PCB for the bracelet with eight actuators. Two of the eight LRAs are shown. D) A closeup of one bracelet LRA placed into an open pill-shaped enclosure.

six 2-channel class-D amplifiers (MAX98306, Maxim Integrated), which are directly connected to the vibrotactile actuators. Class-D drivers are preferable for a low-power system as they have good efficiency; they are usually employed in commercial vibrotactile haptic drivers.

\subsection{Current Sensing}

We added high-speed current sensing on each vibrotactile actuator channel. Two 16:2 multiplexers (MAX14661, Maxim Integrated) allow selecting which channel is connected to the amplifier. For 


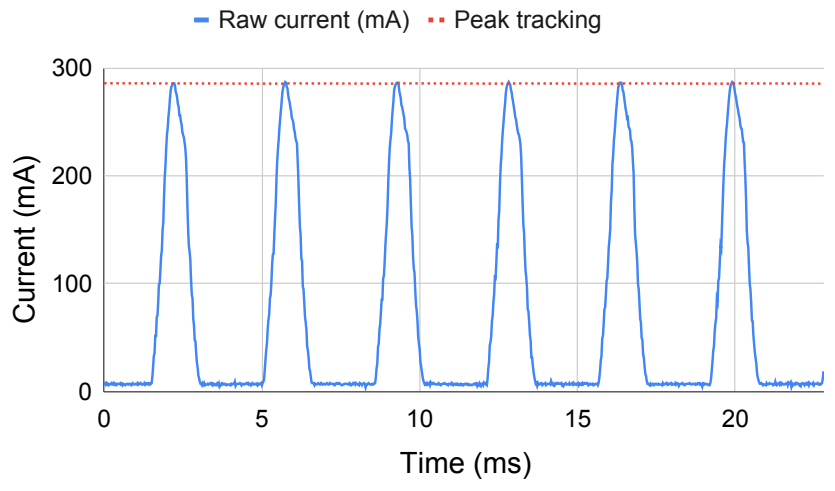

Figure 5: Raw current peak captured from the running actuator with a $250 \mathrm{~Hz}$ sine wave waveform. The data is naturally half-wave rectified as the negative current swings are outside of the current amplifier's range. The dotted red line is the output of the peak-tracking algorithm.

every channel, a $0.25 \Omega$ resistor was placed in series with the positive output to measure the voltage drop due to the current. The resistor was sized to measure the maximum current range. To measure the current, we use a high-bandwidth $1.8 \mathrm{MHz}$ current amplifier (MAX4073, Maxim Integrated) with 20X gain. A bandwidth of $1.8 \mathrm{MHz}$ is needed to properly measure the class-D amplifier's pulse-width modulation output at $540 \mathrm{kHz}$. The current amplifier is followed by an active first-order low pass filter to provide low impedance and anti-aliasing for the analog-to-digital converter (ADC). Only one channel can be connected at a time. Power gating switches were added to completely shut down the current sensing circuit when not in use, as the current amplifier has a high quiescent current (0.5-1.2 mA).

An ADC samples the current at a frequency of $43.215 \mathrm{kHz}$ with a 10-bit resolution. A sample of the typical current usage is shown in Figure 5. The current peak level is tracked using an asymmetric smoothing filter implemented by the microcontroller. The height of the peaks represents the amount of loading for the actuator. The filter output is computed for each new data point as follows:

$$
\begin{aligned}
& K_{\text {attack }}=1-\exp \left(-1 /\left(C_{\text {attack }} f_{S}\right)\right), \\
& K_{\text {decay }}=1-\exp \left(-1 /\left(C_{\text {decay }} f_{S}\right)\right), \\
& K= \begin{cases}K_{\text {attack }} & \text { if } I_{\text {analog }} \geq I_{\text {peak }}[n], \\
K_{\text {decay }} & \text { if } I_{\text {analog }}<I_{\text {peak }}[n],\end{cases} \\
& I_{\text {peak }}[n]=I_{\text {peak }}[n-1]+K \cdot\left(I_{\text {analog }}-I_{\text {peak }}[n-1]\right),
\end{aligned}
$$

where $I_{\text {analog }}$ is the current reading and $I_{\text {analog }}$ is the tracked peak current. $C_{\text {attack }}$ and $C_{\text {decay }}$ are attack and decay time constants, $f_{S}$ is the sample rate, and $n$ is the sample index. We use an attack time of $10 \mathrm{~ms}$ and a decay time of $1.5 \mathrm{sec}$. The slow decay time was used to ensure the filter is functional at low actuation frequencies.

When switching between different actuator channels, the filter needs time to settle. We found that settling time is around $500 \mathrm{~ms}$, thus limiting the sampling rate if multiple channels are measured.

\subsection{Other Sensors}

We integrated several sensors into the board to provide more experimental flexibility. There are two audio options for audio-to-tactile processing. First, an onboard pulse density modulation (PDM) digital microphone (CMM-4030DT, CUI Devices). Second, a $2.5 \mathrm{~mm}$ jack for external analog audio input, conditioned by a microphone amplifier (MAX9814, Maxim Integrated) for the ADC, as the user might position a microphone outside the device. We included a 3-axis low-power accelerometer (LIS3DH, STMicroelectronics) for activity sensing. We use a voltage divider to measure the battery voltage and a tactile button for general purpose use. Finally, a thermistor is a safety mechanism to prevent the vibrotactile amplifiers from overheating.

\subsection{Manufacturing}

We designed the board to be manufacturable in large quantities. The components are widely available. Most of the cost is the components. The current cost of all components is about $\$ 43$ when purchased in quantities of 1000 . At that quantity, the manufacturing cost of each blank board is $\$ 0.52$. Including assembly, the total cost is about $\$ 50$.

To make the board manufacturable, it is necessary to design a fast and robust automated factory-test procedure of the main functionality. All the vibrotactile actuators can be quickly tested by connecting to 12 vibrotactile actuators. Our current-based selftesting procedure was used, without the need for external sensors. We designed a text-based interface that tested all the functionality of the board.

\section{PROGRAMMING INTERFACES}

The code is divided into the firmware, that was optimized to run on the board itself, and software that controls the hardware from another device.

\subsection{Firmware}

The firmware is a portable $\mathrm{C} / \mathrm{C}++$ library that works in Arduino as well as other IDEs, such as Segger Embedded Studio, to enable quick prototyping. We used the existing Adafruit Feather nRF52840 board definition [1], as it provides a USB-serial and Bluetooth bootloader. However, to get the optimal performance we wrote all the drivers on top of the low-level Nordic's HAL (Hardware-Abstraction Layer) [45]

\subsection{Software}

We created a PC application that allows real-time playback of up to 12 waveforms on the haptic actuators. The data is streamed to the VHP over a USB connection, allowing playback of arbitrary length waveform. The waveforms are 16-bit signed integer 2000 $\mathrm{Hz}$ PCM (pulse-code modulation) format. Before a data frame is finished playing, a synchronization packet is sent from the VHP to the PC application, which triggers the next frame to be sent. The bandwidth requirement to stream all channels is $32 \mathrm{kbps}$. The USB connection could be used in two ways:

First, we created a Python app that uses a simple command-line interface to continuously play any 12-channel $2000 \mathrm{~Hz}$ WAV file. 
The haptic waveform WAV files can be created and edited using off-the-shelf software such as Audacity ${ }^{3}$, as shown in Figure 6.

Second, the haptic waveforms can be created computationally using Python, and played back in real-time. We used Jupyter notebooks to create a convenient browser-based user interface. This is useful for creating haptics experiments with user interfaces, as we describe in the applications section.

We don't believe that a custom waveform editing GUI (Graphical User Interface) has to be designed, as existing and well-developed audio-editing software can be used (e.g., Audacity, MAX MSP). Furthermore, audio-editing software does not allow real-time waveform generation based on input sensor data, such as converting audio to tactile in real-time. For such generation, an embedded and highly optimized solution is needed.

Bluetooth 4.2 protocol cannot easily support 12-channel streaming due to bandwidth and latency limitations. However, Bluetooth can be used to trigger waveforms and view and control different operational parameters of the device. Our Javascript browser-based app can run on a PC or a phone with a Bluetooth connection. The interface is shown in Figure 7. Multiple VHP boards can be connected over Bluetooth; however, they should have unique names to be easily identifiable. This facilitates use-cases such as wearing VHP bracelets on the left and right arms simultaneously.

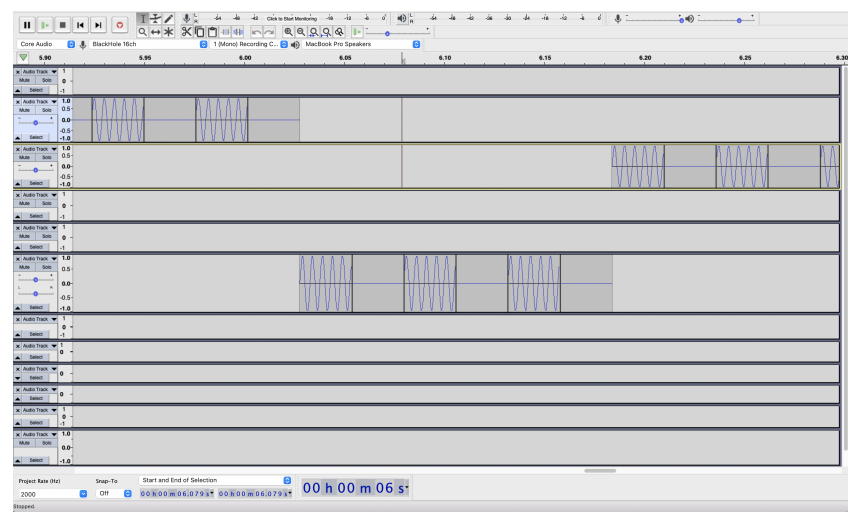

Figure 6: Screenshot of waveforms editing for haptics in Audacity, showing the 12-channel cutaneous-rabbit experiment haptic stimulus.

\section{TECHNICAL EVALUATION}

In this section, we evaluate the important technical parameters. In all tests, except identifying actuator types, we use an LRA actuator (LV101040A, Jinlong Machinery \& Electronics, Inc). We used LRAs for testing since it is the most commonly used vibrotactile actuator for small devices.

\subsection{Power Consumption and Battery Life}

As we expect the device to function on a battery, its power consumption is important. To measure current, we used a multimeter

\footnotetext{
${ }^{3}$ https://www.audacityteam.org/
}

\section{Audio-to-Tactile BLE web client}

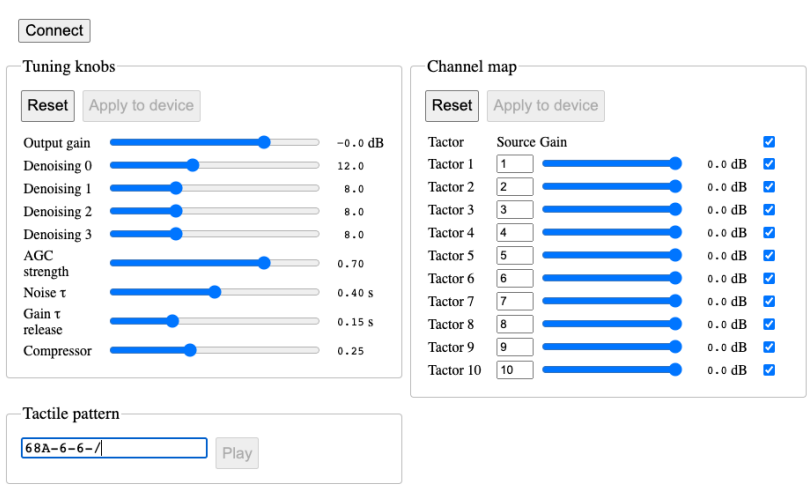

Figure 7: Screenshot of the browser-based interface to control VHP over Bluetooth.

(U1252B, Agilent) and a $3.7 \mathrm{~V}$ supply. We report the mean current consumption over a 5-minute interval, to smooth out fluctuations.

The idle power consumption with Bluetooth connected is $4.8 \mathrm{~mA}$, meaning that the baseline consumption of the VHP will be 104 hours on a $500 \mathrm{mAh}$ battery. Realistically, the power consumption will be higher.

With all six haptic amplifiers and PWM modules on, but driving no actuators, the power consumption is increased to $19.7 \mathrm{~mA}$. Assuming a worst case with one actuator always on, with a full amplitude sine wave $(200 \mathrm{~Hz})$, the current consumption is $183 \mathrm{~mA}$. The $500 \mathrm{mAh}$ battery could last between 3 hours with one actuator always on up to 25 hours with minimal actuator activity. The actual power consumption would vary based on the duty cycle and amplitude of the haptic signals, and will be somewhere in between these times if on average less than one actuator is running at full amplitude.

\subsection{Number of Actuators That Can Be Run Simultaneously.}

Each actuator uses a significant amount of current when at full power, drawing a peak of roughly $460 \mathrm{~mA}$, assuming $8 \Omega$ resistance. Thus, we want to understand how the power supply voltage is affected as multiple actuators are turned on simultaneously.

We measured supply voltage on the board, and turned on actuators, increasing the number by one every 10 seconds. We used a full-amplitude sine wave at $200 \mathrm{~Hz}$. This test was conducted with a bench power supply (SPD1168X, Siglent) and a $500 \mathrm{mAh}$ lithiumpolymer battery (ASR00035, TinyCircuits). The battery was fully charged to $4.2 \mathrm{~V}$, and the bench supply was set to $4.2 \mathrm{~V}$ as well.

Figure 8 shows the current requirements. We measured the maximum current draw at about 2.1 A. We determined that when batterypowered, the board can support all 12 actuators. However, the power regulation can become unreliable if more than nine actuators are active; thus, this is not recommended. The low-drop-out regulator (LDO) used to regulate the battery voltage to a stable $3.3 \mathrm{~V}$ needs about $100 \mathrm{mV}$ overhead; thus needing an input voltage above $3.4 \mathrm{~V}$. 


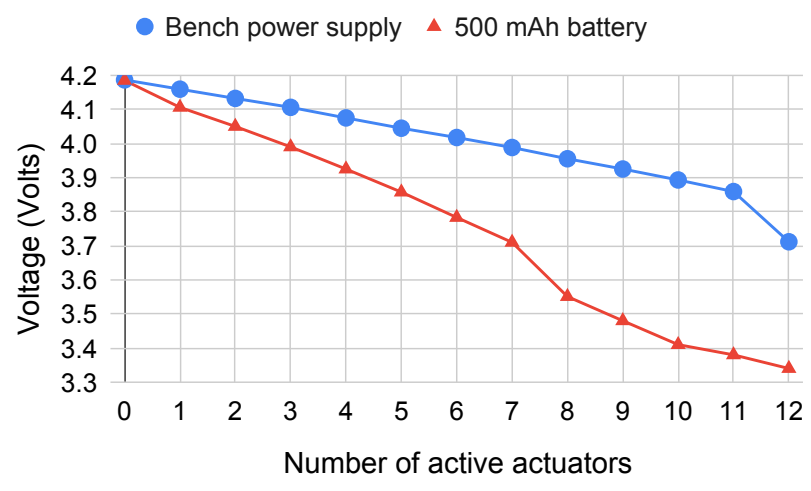

Figure 8: Graph showing rail voltage drop as multiple actuators are turned on simultaneously. The voltage measurement was done on the VHP board with both a battery and a bench power supply. The bench power supply voltage drop was due to the resistance of the wires and the PCB traces. The system can handle a voltage drop to around 3.4 V.

A higher-capacity battery with 1000 to $1500 \mathrm{mAh}$ should be able to handle 2 A continuous without significant voltage drops. A $3.7 \mathrm{~V}$ bench power supply can support all 12 actuators.

\subsection{Latency}

Latency is an important parameter to minimize when driving tactile actuators. The maximum latency in the hardware update loop is $4 \mathrm{~ms}$, as the PWM buffer can be updated with new data right before it finishes playing the previous buffer. The buffer was optimized to have $4 \mathrm{~ms}$ of data. However, the latency is increased with communications. Assuming the worst-case end-to-end scenario, when sending data from a PC (2020 MacBook Pro, Apple) to the actuator the latency is $7 \mathrm{~ms}$. This latency is from when the key is pressed on the keyboard to the time the waveform is modulated on the actuator. The measurement was taken using the oscilloscope (MSOX2024A, Keysight), with one lead connected to a tactile button placed on the computer key and another to the PWM output of the VHP board. This latency is difficult to reduce, as it includes the operating system latency.

\subsection{Sensing the Actuator Loading Current}

In this section, we describe tests to quantify the load-current sensing. We place the vibrotactile actuator on a foam pad to decouple any external vibrations. The vibration frequency is swept from 0 to $400 \mathrm{~Hz}$ during a 30-second measurement to quantify the sensor's full range and to better understand the complex current-frequency relationship.

First, we do the measurement with no mechanical loading. Then, we load the actuator with a light fingertip touch. Finally, we press on the actuator with a heavy touch. The differences between those three conditions are seen in Figure 9A. The most pronounced difference is around 230 to $270 \mathrm{~Hz}$, most likely due to the mechanical construction of the actuator, and would be different with another actuator. In this range, the loading increases current consumption.
However, in other frequencies, the relationship can be more complex. Also, the measurements show that the sensor does not operate in the whole 0 to $400 \mathrm{~Hz}$ range, as the current drops to close to zero on both ends. The reliable driving range is about 40 to $350 \mathrm{~Hz}$.

After analyzing the frequency sweep data, we picked a single frequency of $250 \mathrm{~Hz}$ for sensing. We wanted to see if this technique worked in practice. We used the LRA in the bracelet form factor, as described in subsection 7.3. The bracelet was tightened and loosened around the wrist. In Figure 9A, the current clearly increased when the bracelet strap is tightened, as it increases contact pressure between the skin and LRA. The increased load on the motor causes a measurable increase in current.

\subsection{Using Current to Identify Actuator Types}

There are a large variety of vibrotactile actuators, and each one can behave differently and thus might need different presets. Thus, we believe that the ability to identify the actuator automatically can make the platform more usable. We collected current-load data from three commonly available but different actuators, as can be seen in Figure 4C:

- LRA (LV101040A, Jinlong Machinery \& Electronics, Inc),

- Voice coil (BHS16-11P-08H5.8W30, BeStar Technologies, Inc.)

- Linear Magnetic Ram (TH-153308-S, Nanoport Technology Inc.)

The current was measured and binned at thirty intervals with a $31 \mathrm{~Hz}$ increment, from 31 to $938 \mathrm{~Hz}$. The frequency sweep was repeated five times for each actuator, and we also collected data from three different actuators of the same type. In total we collected data from $5 \times 3 \times 3=45$ runs. During testing, actuators were placed on a foam pad.

We trained a simple support vector machine (SVM) classifier using the scikit-learn library [36] with linear kernel, using a 70/30 train/test data split. The test accuracy was $100 \%$ for classifying the three classes, indicating that each actuator has a very distinct response, as shown in Figure 10. With only three distinct actuators, a simple classifier can identify actuators. However, if more models and types of actuators are added, a more sophisticated classier might be needed.

\subsection{Comparison with the Back-EMF Sensing}

We compare the current load measurement technique with a backEMF approach [9]. This prior work sensed only one channel, so for this comparison, it was scaled to 12 channels by increasing the number of analog switches and adding a multiplexer.

The key comparison parameters are shown in Table 1 . The advantages of current load sensing are a lower footprint, power consumption, and the ability to sense and actuate simultaneously. The disadvantages include a slower sampling rate, no ability to sense passively and imperceptibly.

\section{APPLICATIONS}

In this section, we show how the platform can be directly integrated into various form factors to make fully wearable devices. We show an audio-to-tactile application of the bracelet that was was worn 

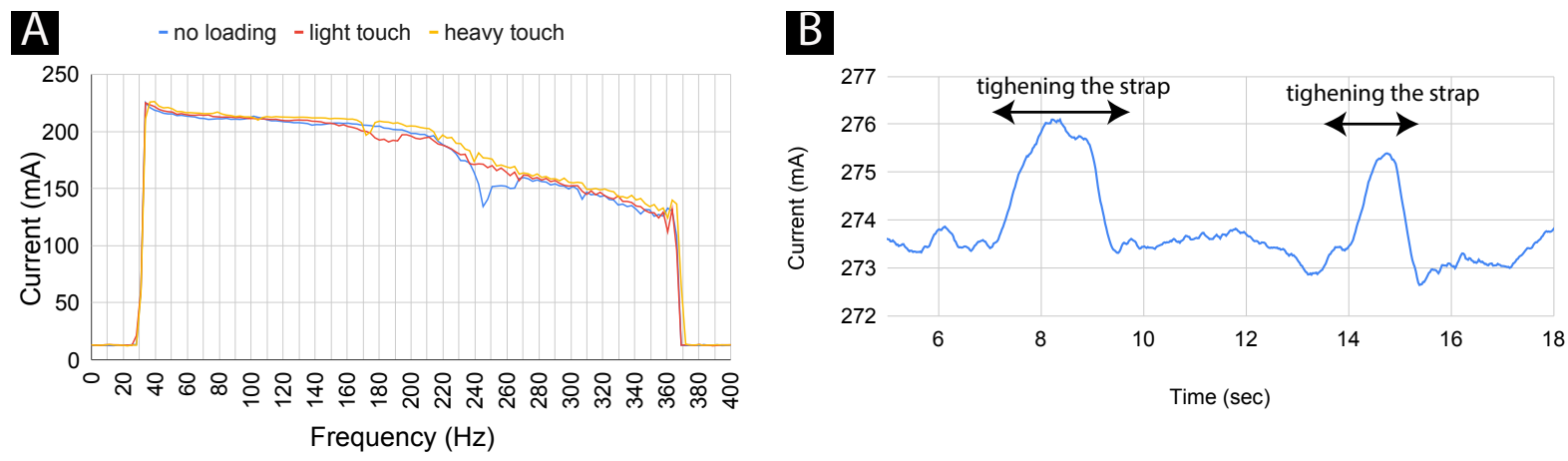

Figure 9: Load sensing. A) Graph showing filtered peak current measurements of the LRA during a frequency sweep from 0 to $400 \mathrm{~Hz}$. Three conditions were tested: no loading, light and heavy loading with a fingertip. B) Data were taken from an LRA inside a bracelet form-factor, when the strap is tightened (increased skin contact) for a few seconds at a $250 \mathrm{~Hz}$ vibration frequency. The shown data is filtered with a moving average of 20 samples

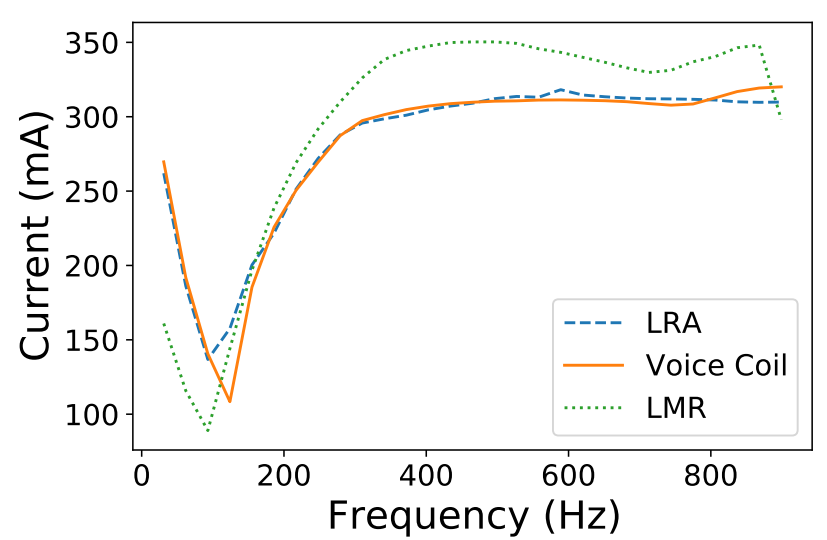

Figure 10: Overlay of filtered peak current measurements of three actuators, demonstrating unique responses of each. Samples were measured at $31 \mathrm{~Hz}$ to $938 \mathrm{~Hz}$ frequencies, at 31 Hz intervals.

Table 1: Comparison of key parameters of loading current sensing (this work) and back-EMF sensing.

\begin{tabular}{|l|l|l|}
\hline & Back-EMF & This work \\
\hline Number of chips & 27 & 4 \\
\hline Quiscent power consumption & $4.38 \mathrm{~mA}$ & $0.63 \mathrm{~mA}$ \\
\hline Sampling rate each channel & $55 \mathrm{~Hz}$ & $2 \mathrm{~Hz}$ \\
\hline Actuator frequency range & sub Hz $-380 \mathrm{~Hz}$ & $40-350 \mathrm{~Hz}$ \\
\hline Imperceptible sensing & yes & no \\
\hline Passive sensing & yes & no \\
\hline Simultaneous sensing/actuation & no & yes \\
\hline
\end{tabular}

for an extended period of by the authors. Also, we show how the system can be applied to psychophysical tests.

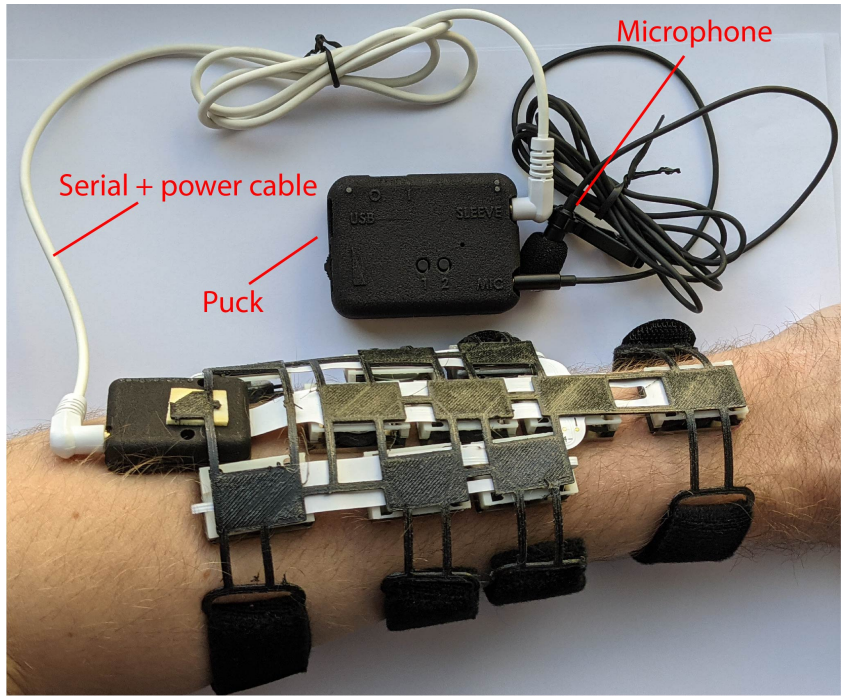

Figure 11: Sleeve with ten vibrotactile actuators and VHP board. The sleeve is controlled via serial communications and powered with a custom designed "puck" device, over the white audio cable.

\subsection{Form Factors}

VHP allows quick integration into various form factors. We integrated the VHP into three different form factors: sleeve, bracelet, and mobile phone case. The sleeve is more suitable for tethered experimentation, while the bracelet can be used in wearable everyday scenarios. The development did not require any specialized equipment except for a 3D printer.

The sleeve was 3D printed with PETG (3D PETG-1KG1.75-ORN, HATCHBOX) and flexible TPU (3DAR0117505, NinjaTek) materials, and voice coils (12041808, Soberton Inc.) were used as vibrotactile actuators. We designed a flex PCB to connect all the vibrotactile 

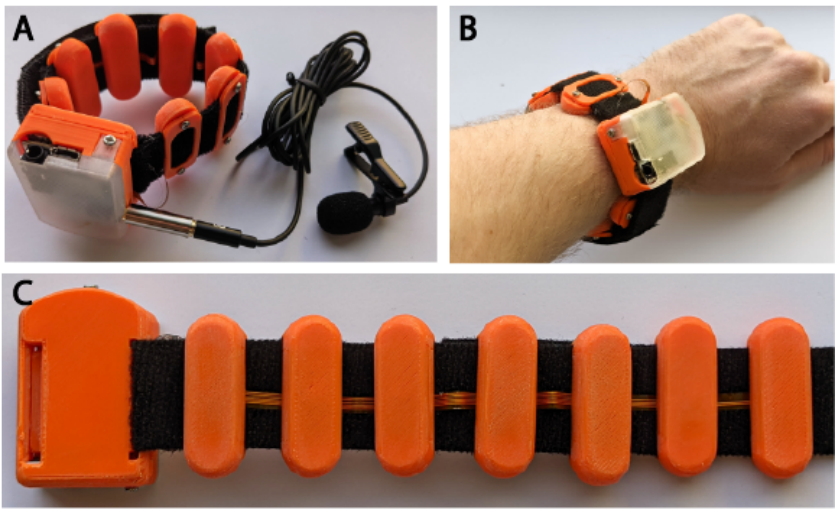

Figure 12: Integrating the VHP platform into an 8-actuator bracelet for audio-to-tactile applications. A) The bracelet is connected to an external microphone with $2.5 \mathrm{~mm}$ to $3.5 \mathrm{~mm}$ audio jack adapter. The large box contains the battery, the VHP board and an actuator. Each pill-shaped box contains a linear resonant actuator. B) Bracelet on the arm. C) The backside of the bracelet. The FPC, which connects actuators to the mainboard, is running horizontally in the middle.

actuators. The VHP board on the sleeve is tethered to a second custom-designed "puck" with a $3.5 \mathrm{~mm}$ audio jack for power and serial data, as shown in Figure 11. This additional device was designed to be carried in a pocket (allowing a larger battery than could be worn on the sleeve) and to support buttons and switches as well as on-board and off-board microphones and a Bluetooth control interface.

As we found the sleeve form factor to be inconvenient for daily wear, we constructed a smaller device with an integrated battery, that does not use the puck. The bracelet was constructed using PEG 3D printed material and Velcro, as shown in Figure 12. We used wideband LRAs (LV101040A, Jinlong Machinery\& Electronics, Inc.) as the vibrotactile actuators, which were taped into the pillshaped boxes. The bracelet was self-contained and powered with a $500 \mathrm{mAh}$ lithium-polymer battery. The design required a custom FPC and 3D-printed designs. The spacing of actuators can be easily adjusted by loosening the screws.

Besides wearable uses, the platform can be integrated into small devices. As mobile phones have only one or two actuators, expanding the number of actuators could be useful to provide additional tactile information. This is especially useful as fingers are sensitive to vibrations. As a result, we created a phone-case prototype for a mobile phone (Pixel 4, Google) with eight vibrotactile actuators. The prototype is shown in Figure 13. The case was custom-designed and 3D printed. We also added a telescopic grip, so the phone can be held between two fingers, maximizing contact with the actuators. The VHP circuit board was placed inside the phone case, allowing control of eight actuators on the case.

\subsection{Psychophysical Tests}

We test the sleeve's functionality and performance using simple psychophysical tests. We use Jupyter ${ }^{4}$ as a front end because it

\footnotetext{
${ }^{4}$ https://jupyter.org/
}

allows quick iteration and experimentation. We describe two simple experiments here: perceptual thresholds and spatial masking. The goal of these experiments is to demonstrate that the platform can be used for common psychophysical studies and and not to draw new conclusions.

In the first experiment, we measure the perceptual threshold across frequency for a single voice coil actuator. We use a Levitt 2-down-1-up paradigm [28] to find the point where the subject is $71 \%$ accurate in a two-alternative forced-choice (2AFC) experiment. The subject was cued with audio to delineate the two intervals (500 $\mathrm{ms}$ each) in which a sinusoid with a frequency between 62.5 and 500 $\mathrm{Hz}$ and a Hanning envelope window might be present. Figure 14 shows a snippet of the Jupyter notebook that defines the test and provides an elementary GUI. The results for one subject are shown in Figure 14. This is uncalibrated, so the levels are relative, but does show the best threshold around $250 \mathrm{~Hz}$, similar to that described in the literature [6].

In the second experiment, we measured spatial masking, the threshold of a probe actuator as a function of the distance to a masking actuator, along the forearm of a subject. Like above, a $500 \mathrm{~ms}$ sinusoid at $125 \mathrm{~Hz}$ was played in one actuator during both intervals. In one of the intervals an additional probe sinusoid was played with varying amplitude to a different actuator. The subject had to specify which interval contained the two simultaneous sinusoids. The results in Figure 14 show a high threshold when the masker is closest to the probe actuator, and then better (lower) thresholds as the masking actuator moves further away, as shown by Sherrick [46]. This type of experiment is useful as we explore multi-actuator signal representations.

\subsection{Audio-to-Tactile Bracelet for Everyday Wear}

We used previously developed algorithms for real-time audio-totactile conversion, available via GitHub [19], and ran them on the VHP's nRF52 microcontroller. The algorithms included filter banks and neural networks for fricative and vowel representation. To capture surrounding sound, an external omnidirectional microphone (Model\#4330236175, Miracle Sound), was plugged into the $2.5 \mathrm{~mm}$ audio jack of the bracelet. Below, we highlight the challenges and insights from wearing the audio-to-tactile bracelet daily for several months by one of the developers, who will be referred to as the "target user". This user employs lipreading in everyday life and has significant long-term experience with various audio-to-tactile wearable devices. The bracelet has also been worn for shorter periods (days) by other developers.

7.3.1 Hardware usability in everyday use. All the developers found the bracelet to be reliable and comfortable to wear throughout the day. The battery life was 13 hours in a reasonably quiet workingfrom-home environment with occasional online meetings. Typically the bracelet was left to charge overnight and worn during the day without additional charging.

The PETG 3D-printed enclosure worked well, indicating that an inexpensive home $3 \mathrm{D}$ printer is practical. However, several of the strap holders on the enclosure broke after a few months and needed repair. Thus, we recommend injection molding or more robust 3Dprinted Nylon using an industrial Selective Laser Sintering (SLS) printer for long-term wear. 

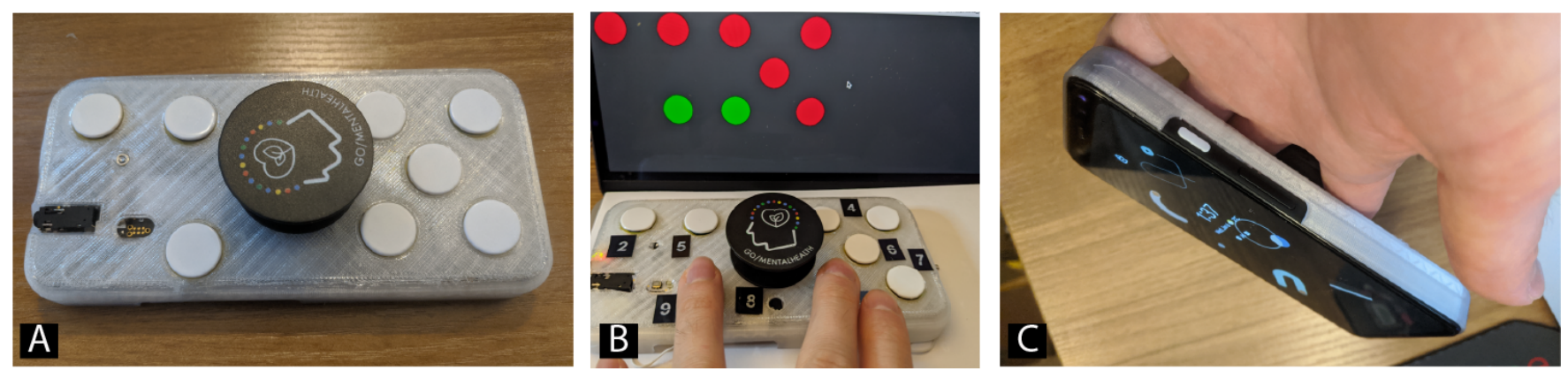

Figure 13: A) 3D-printed mobile phone case with eight voice coil actuators, as seen as white circles. The VHP is integrated inside the case, and can be powered by a battery or through an audio jack. B) The visualization showing which actuators are touched by fingers, as displayed by green circles. C) Phone case can be held between the fingers.
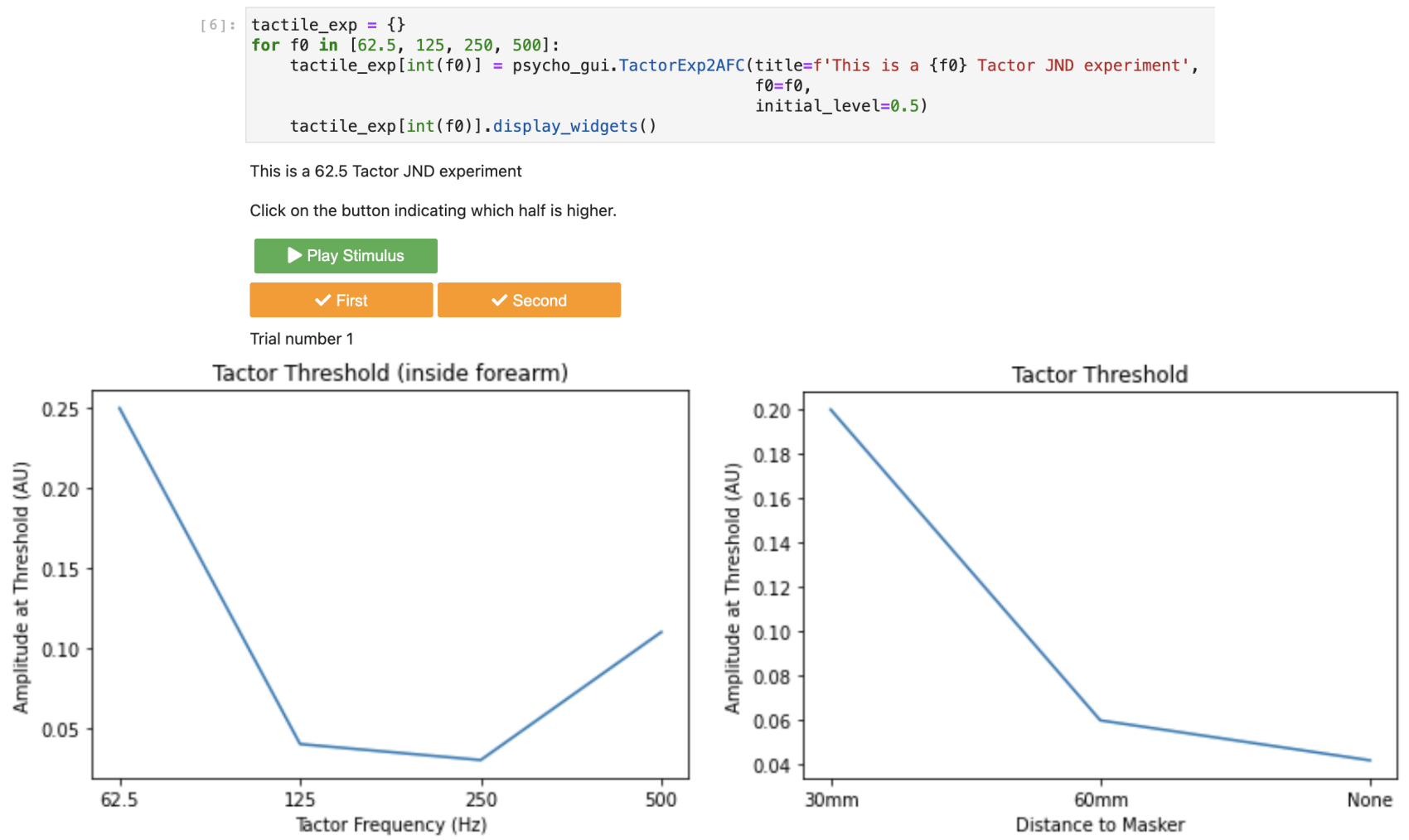

Figure 14: Top: A section of a Jupyter notebook that we use for testing. Bottom left: The perceptual threshold for one subject as a function of frequency. Bottom right: The results of a spatial-masking experiment, showing better (lower) thresholds further from the masker. Note, these are pilot experiments to show that we can do these experiments using VHP. More careful experiments will follow in a separate paper. "Tactor" refers to the voice coil actuator.

An unexpected challenge was using an external analog microphone, highlighting the usefulness of open source hardware, as it allows quick modifications not initially envisioned by the platform. We tested various microphones attached directly to the bracelet or wire to be clipped somewhere on the clothing (As seen in Figure 12A). We found both attachments to work well; however, the microphone directly on the bracelet was more convenient. We employed the $2.5 \mathrm{~mm}$ audio jack for the microphone; however, all the off-the-shelf microphones were $3.5 \mathrm{~mm}$, thus we needed a bulky adapter. The microphone stuck out on the side with the adapter, thus making it awkward to use with sleeves. We used the second audio jack, initially intended as a serial port, to plug in the microphone 
directly. Thus, pointing the microphone towards the fingers. We found that with the microphone pointing towards fingers, the target user sometimes moved the wrist to localize the position of the sound source. The closer hand got to the source, the stronger the haptic actuators become.

7.3.2 Lip-reading aid impressions. The impression of our target user was that his lipreading in combination with haptics devices improved. This user found that our wearable haptic bracelet gives better information to facilitate lipreading than previous devices. Specifically, the target user improved understanding via lipreading some words that were difficult to understand only via lipreading (phonemic contrasts like "pin" vs. "min") but could be easily recognized when wearing the bracelet. The user felt that some different sound classes (like some consonants) produced a characteristic vibration pattern that could be learned. The user also found it helpful to wear two bracelets (on the left and right wrists) and configured the sound-to-haptics mapping differently for each hand. More testing is needed to validate these observations.

Ambient noise became a problem when wearing the device around the house. Background noise such as a fan would cause the actuators to be continuously buzzing, which annoyed the users. So we developed an adaptive noise canceling technique to alleviate this issue.

7.3.3 Software functionality. The Arduino programming environment allowed one to quickly change and share the embedded code. Initially, we used embedded systems-specific tools such as Segger Embedded Studio ${ }^{5}$ and Makefiles but found them difficult for unfamiliar users. For example, the target user could try different haptic channel mappings at home quickly, without previous embedded programming experience. We found this to be essential for development. For example, the user discovered it useful to reassign fricative-range energy (above $2500 \mathrm{~Hz}$ ) to an actuator farther away in the bracelet from an actuator displaying lower-frequency energy (below $500 \mathrm{~Hz}$ ). When actuators are close on the skin, one can confuse which one is associated with a given stimulus.

\subsection{Adjustable Haptics on Phone Case}

A phone case provides a lot of unused real estate for haptics. This is especially important as the sensitive fingertips are usually positioned on the back of the phone. Putting so many vibrotactile actuators on the back of the phone provides some challenges. For one, our preferred grips are different, and not all fingers will touch all actuators. We need to know which actuators are used, and turn off the rest. This is done to conserve the energy on a small mobile device. Thus, we can use our load estimation ability to automatically sense which actuators are being used. The finger contact was measured on each actuator in round-robin fashion, with each measurement taking $1.7 \mathrm{sec}$, and taking the mean of 50 readings. Finger contact was detected if the value was above a threshold Such actuator adjustments would not be possible without using the VHP.

When holding the phone with both hands in landscape mode, the case can provide left and right-side haptic feedback separately. The VHP board in the case can be connected to the phone over

\footnotetext{
${ }^{5}$ https://www.segger.com/products/development-tools/embedded-studio/
}

Bluetooth. This could be used to enhance effects in a mobile game or when watching a video. The moving part of voice coil actuators are not coupled to the phone's chassis, thus providing higher bandwidth and more intense sensation. Also, the case can provide rich notifications, especially when the display is not accessible, such as when the phone is in the pocket.

\section{LIMITATIONS AND FUTURE WORK}

This paper presents pilot experiments that show that VHP can be used as a wearable audio-to-tactile device. We saw promising results, especially as a lipreading aid. In the future, we plan to conduct full-scale experiments with multiple users wearing the device for an extended time.

To date, we have demonstrated a small number of applications using our platform. To fully understand the usability and uses of our platform, we plan to conduct long-term user studies such as workshops with diverse groups of users with different skill levels.

To best integrate the VHP platform in a miniaturized device, in many cases, still requires making a custom flexible circuit board and soldering skills. This is a barrier to entry for those with limited electronics skills. There is no simple template as it is challenging to accommodate all the various shapes of devices and actuators. We plan to explore how we can make a standard flexible PCB that can easily integrate and fit the most common uses. Also, making integrated prototypes still requires 3D modeling skills and 3D printer access. To alleviate this, we plan to open-source the mechanical design of our existing haptic devices, such as the bracelet. Such designs could be easily adapted without 3D modeling skills and can be made using a standard 3D printer. Furthermore, we hope that the community will create and share additional designs that can be adapted.

\section{CONCLUSION}

In this paper, we introduce VHP, a platform for wearable haptic interfaces. The platform was based on our research needs towards more wearable haptics and met our initial design criteria, such as small, low power, and low latency. At its core, the platform comprises a small PCB that can output twelve-channel vibrotactile signals with a latency of 4-7 ms. The platform allows for USB, serial, and Bluetooth communications. VHP also has sensing abilities such as external and internal microphones and accelerometers. Furthermore, the actuators can sense loading to allow for consistent contact with the skin. The device can be integrated directly into a wearable device and can provide all-day battery life, which, to the best of our knowledge, is not offered by other platforms. We provide easily accessible and flexible open-source software and firmware. We also plan to partner with distributors and manufacturers to make the hardware available.

We demonstrated several practical applications using the VHP. We demonstrate how VHP can be used in an audio-to-tactile wearable device. VHP was integrated into a bracelet and a forearm sleeve to provide a convenient and daily wearable audio-to-tactile interface. This demonstrates potential future use as a wearable sensory substitution and aid for lipreading. We also show how VHP was integrated into a mobile phone case to expand the haptics of our gadgets. Finally, we demonstrate how VHP and its interfaces can 
be conveniently and quickly used in traditional psychophysical haptics experiments such as JND (just noticeable difference), by conveniently connecting to a browser-based user interface.

We believe that the progress of haptics development is hindered by the lack of available platforms. Current large lab-based setups prevent the use and experimentation with haptics in everyday life. After all, tactile is one of the fundamental human senses but has been overlooked in favor of audio and visual technology. We hope that with our platform, we can help democratize the use of haptics and inspire a more widespread use of tactile devices.

\section{ACKNOWLEDGMENTS}

We thank Alex Olwal, Thad Starner, Hong Tan, Charlotte Reed, Sarah Sterman, Yuhui Zhao, Dmitrii Votintcev, Chet Gnegy, Whitney Bai and Sagar Savla.

\section{REFERENCES}

[1] Adafruit. 2021. Arduino Core for Adafruit Bluefruit nRF52 Boards. https://github. com/adafruit/Adafruit_nRF52_Arduino. Accessed 2021-03-14.

[2] Apple. 2021. Core Haptics. https://developer.apple.com/documentation/ corehaptics. Accessed 2021-03-08.

[3] Yasuyoshi Asai, Katsuhiro Hirata, and Tomohiro Ota. 2013. Amplitude control method of linear resonant actuator by load estimation from the back-EMF. IEEE transactions on magnetics 49, 5 (2013), 2253-2256.

[4] Mojtaba Azadi and Lynette A Jones. 2014. Vibrotactile actuators: Effect of load and body site on performance. In 2014 IEEE Haptics Symposium (HAPTICS). IEEE, 351-356.

[5] bHaptics. 2021. TactSuit X40. https://www.bhaptics.com/tactsuit/tactsuit-x40 Accessed 2021-03-01.

[6] S. J. Bolanowski, G. A. Gescheider, R. T. Verrillo, and C. M. Checkosky. 1988. Four channels mediate the mechanical aspects of touch. The fournal of the Acoustical Society of America 84, 5 (1988), 1680-1694. https://doi.org/10.1121/1.397184 arXiv:https://doi.org/10.1121/1.397184

[7] Aditya Tirumala Bukkapatnam, Philippe Depalle, and Marcelo M Wanderley. 2020 Defining a vibrotactile toolkit for digital musical instruments: characterizing voice coil actuators, effects of loading, and equalization of the frequency response. fournal on Multimodal User Interfaces 14, 3 (2020), 285-301.

[8] Roger W Cholewiak and Carl E Sherrick. 1986. Tracking skill of a deaf person with long-term tactile aid experience: a case study. Rehabilitation Research and Development Service.

[9] Artem Dementyev, Alex Olwal, and Richard F. Lyon. 2020. Haptics with In put: Back-EMF in Linear Resonant Actuators to Enable Touch, Pressure and Environmental Awareness. In Proceedings of the 33rd Annual ACM Symposium on User Interface Software and Technology (Virtual Event, USA) (UIST '20). Association for Computing Machinery, New York, NY, USA, 420-429. https //doi.org/10.1145/3379337.3415823

[10] Android Developers. 2021. VibrationEffect. https://developer.android.com/ reference/android/os/VibrationEffect. Accessed 2021-03-08.

[11] Jan BF Van Erp, Hendrik AHC Van Veen, Chris Jansen, and Trevor Dobbins. 2005 Waypoint navigation with a vibrotactile waist belt. ACM Transactions on Applied Perception (TAP) 2, 2 (2005), 106-117.

[12] Mark D Fletcher. 2020. Using haptic stimulation to enhance auditory perception in hearing-impaired listeners. Expert Review of Medical Devices (2020), 1-12.

[13] German Flores, Sri Kurniawan, Roberto Manduchi, Eric Martinson, Lourdes M Morales, and Emrah Akin Sisbot. 2015. Vibrotactile guidance for wayfinding of blind walkers. IEEE transactions on haptics 8, 3 (2015), 306-317.

[14] Jonas Forsslund, Michael Yip, and Eva-Lotta Sallnäs. 2015. WoodenHaptics: A Starting Kit for Crafting Force-Reflecting Spatial Haptic Devices. In Proceedings of the Ninth International Conference on Tangible, Embedded, and Embodied Interaction (Stanford, California, USA) (TEI '15). Association for Computing Machinery, New York, NY, USA, 133-140. https://doi.org/10.1145/2677199.2680595

[15] Karyn L Galvin, Gina Mavrias, Alessandra Moore, Robert SC Cowan, Peter J Blamey, and Graeme M Clark. 1999. A comparison of tactaid ii and tactaid 7 use by adults with a profound hearing impairment. Ear and hearing 20, 6 (1999), 471-482.

[16] Frank A Geldard and Carl E Sherrick. 1972. The cutaneous" rabbit": a perceptual illusion. Science 178, 4057 (1972), 178-179.

[17] Mayank Goel, Jacob Wobbrock, and Shwetak Patel. 2012. GripSense: Using Builtin Sensors to Detect Hand Posture and Pressure on Commodity Mobile Phones In Proceedings of the 25th Annual ACM Symposium on User Interface Software and Technology (Cambridge, Massachusetts, USA) (UIST '12). Association for
Computing Machinery, New York, NY, USA, 545-554. https://doi.org/10.1145/ 2380116.2380184

[18] Steven Goodman, Susanne Kirchner, Rose Guttman, Dhruv Jain, Jon Froehlich, and Leah Findlater. 2020. Evaluating Smartwatch-Based Sound Feedback for Deaf and Hard-of-Hearing Users Across Contexts. In Proceedings of the 2020 CHI Conference on Human Factors in Computing Systems (Honolulu, HI, USA) (CHI '20). Association for Computing Machinery, New York, NY, USA, 1-13. https://doi.org/10.1145/3313831.3376406

[19] Google. 2021. Audio-to-Tactile Representation Boards. https://github.com/google/ audio-to-tactile. Accessed 2021-03-29.

[20] Ian Hattwick, Ivan Franco, and Marcelo M Wanderley. 2017. The vibropixels: A scalable wireless tactile display system. In International Conference on Human Interface and the Management of Information. Springer, 517-528.

[21] Sungjae Hwang, Andrea Bianchi, and Kwang-yun Wohn. 2013. VibPress: Estimating Pressure Input Using Vibration Absorption on Mobile Devices. In Proceedings of the 15th International Conference on Human-Computer Interaction with Mobile Devices and Services (Munich, Germany) (MobileHCI '13). Association for Computing Machinery, New York, NY, USA, 31-34. https: //doi.org/10.1145/2493190.2493193

[22] Texas Instruments. 2021. DRV2605L: Haptic driver for ERM/LRA with 2-V operation, waveform library \& auto-resonance tracking. https://www.ti.com/product/ DRV2605L. Accessed 2021-03-01.

[23] Ali Israr, Siyan Zhao, Zachary Schwemler, and Adam Fritz. 2019. Stereohaptics toolkit for dynamic tactile experiences. In International Conference on HumanComputer Interaction. Springer, 217-232.

[24] Lynette A Jones and Nadine B Sarter. 2008. Tactile displays: Guidance for their design and application. Human factors 50, 1 (2008), 90-111.

[25] Jaeyeon Lee and Geehyuk Lee. 2016. Designing a Non-Contact Wearable Tactile Display Using Airflows. In Proceedings of the 29th Annual Symposium on User Interface Software and Technology (Tokyo, Japan) (UIST '16). Association for Computing Machinery, New York, NY, USA, 183-194. https://doi.org/10.1145/ 2984511.2984583

[26] Seungyon Claire Lee and Thad Starner. 2008. Stop burdening your eyes: A wearable electro-tactile display. In 2008 12th IEEE International Symposium on Wearable Computers. IEEE, 115-116.

[27] Seungyon "Claire" Lee and Thad Starner. 2010. BuzzWear: Alert Perception in Wearable Tactile Displays on the Wrist. In Proceedings of the SIGCHI Conference on Human Factors in Computing Systems (Atlanta, Georgia, USA) (CHI '10). Association for Computing Machinery, New York, NY, USA, 433-442. https://doi.org/10.1145/1753326.1753392

[28] H. Levitt. 1971. Transformed Up-Down Methods in Psychoacoustics. The fournal of the Acoustical Society of America 49, 2B (1971), 467-477. https://doi.org/10. 1121/1.1912375 arXiv:https://doi.org/10.1121/1.1912375

[29] Lofelt. 2021. Lofelt Studio. https://lofelt.com/. Accessed 2021-03-08.

[30] Steve Mann, Jason Huang, Ryan Janzen, Raymond Lo, Valmiki Rampersad, Alexander Chen, and Taqveer Doha. 2011. Blind Navigation with a Wearable Range Camera and Vibrotactile Helmet. In Proceedings of the 19th ACM International Conference on Multimedia (Scottsdale, Arizona, USA) (MM '11). Association for Computing Machinery, New York, NY, USA, 1325-1328. https: //doi.org/10.1145/2072298.2072005

[31] Jonatan Martínez, Arturo S García, Miguel Oliver, José P Molina, and Pascual González. 2014. Vitaki: a vibrotactile prototyping toolkit for virtual reality and video games. International fournal of Human-Computer Interaction 30, 11 (2014), 855-871.

[32] Kouta Minamizawa, Yasuaki Kakehi, Masashi Nakatani, Soichiro Mihara, and Susumu Tachi. 2012. TECHTILE toolkit: a prototyping tool for design and education of haptic media. In Proceedings of the 2012 Virtual Reality International Conference. 1-2.

[33] Ryosuke Nakayama, Ryo Suzuki, Satoshi Nakamaru, Ryuma Niiyama, Yoshihiro Kawahara, and Yasuaki Kakehi. 2019. MorphIO: Entirely Soft Sensing and Actuation Modules for Programming Shape Changes through Tangible Interaction. In Proceedings of the 2019 on Designing Interactive Systems Conference. 975-986.

[34] Jifei Ou, Felix Heibeck, and Hiroshi Ishii. 2016. TEI 2016 Studio: Inflated Curiosity. In Proceedings of the TEI'16: Tenth International Conference on Tangible, Embedded, and Embodied Interaction. 766-769.

[35] Sabrina Panëels, Margarita Anastassova, and Lucie Brunet. 2013. TactiPEd: Easy prototyping of tactile patterns. In IFIP Conference on Human-Computer Interaction. Springer, 228-245.

[36] Fabian Pedregosa, Gaël Varoquaux, Alexandre Gramfort, Vincent Michel, Bertrand Thirion, Olivier Grisel, Mathieu Blondel, Peter Prettenhofer, Ron Weiss, Vincent Dubourg, et al. 2011. Scikit-learn: Machine learning in Python. the Journal of machine Learning research 12 (2011), 2825-2830.

[37] Roshan Lalintha Peiris, Wei Peng, Zikun Chen, Liwei Chan, and Kouta Minamizawa. 2017. ThermoVR: Exploring Integrated Thermal Haptic Feedback with Head Mounted Displays. In Proceedings of the 2017 CHI Conference on Human Factors in Computing Systems (Denver, Colorado, USA) (CHI '17). Association for Computing Machinery, New York, NY, USA, 5452-5456. https: //doi.org/10.1145/3025453.3025824 
[38] Michael V Perrotta, Thorhildur Asgeirsdottir, and David M Eagleman. 2021 Deciphering Sounds Through Patterns of Vibration on the Skin. Neuroscience 458 (2021), 77-86.

[39] E. Pezent, B. Cambio, and M. K. O'Malley. 2020. Syntacts: Open-Source Software and Hardware for Audio-Controlled Haptics. IEEE Transactions on Haptics (2020).

[40] Ivan Poupyrev, Shigeaki Maruyama, and Jun Rekimoto. 2002. Ambient Touch Designing Tactile Interfaces for Handheld Devices. In Proceedings of the 15th Annual ACM Symposium on User Interface Software and Technology (Paris, France) (UIST '02). Association for Computing Machinery, New York, NY, USA, 51-60. https://doi.org/10.1145/571985.571993

[41] Jie Oi, Andrew "bunnie" Huang, and Joseph Paradiso. 2015. Crafting Technology with Circuit Stickers. In Proceedings of the 14th International Conference on Interaction Design and Children (Boston, Massachusetts) (IDC '15). Association for Computing Machinery, New York, NY, USA, 438-441. https: //doi.org/10.1145/2771839.2771873

[42] Charlotte M Reed, Hong Z Tan, Zachary D Perez, E Courtenay Wilson, Frederico M Severgnini, Jaehong Jung, Juan S Martinez, Yang Jiao, Ali Israr, Frances Lau, et al. 2018. A phonemic-based tactile display for speech communication IEEE transactions on haptics 12,1 (2018), 2-17.

[43] Robotis. 2021. DYNAMIXEL MX-106R. https://www.robotis.us/dynamixel-mx106r/. Accessed 2021-03-17.

[44] Katsunari Sato, Hiroyuki Kajimoto, Naoki Kawakami, and Susumu Tachi. 2007. Electrotactile Display for Integration with Kinesthetic Display. In RO-MAN 2007 - The 16th IEEE International Symposium on Robot and Human Interactive Communication. 3-8. https://doi.org/10.1109/ROMAN.2007.4415044

[45] Nordic Semiconductor. 2021. Infocenter. https://infocenter.nordicsemi.com/index. jsp. Accessed 2021-03-14.
[46] Carl E. Sherrick. 1964. Effects of Double Simultaneous Stimulation of the Skin. The American fournal of Psychology 77, 1 (1964), 42-53. http://www.jstor.org/ stable/1419270

[47] Sparkfun. 2021. Haptic Motor Driver - DRV2605L. https://www.sparkfun.com/ products/14538. Accessed 2021-03-01.

[48] SUBPAC. 2021. SUBPAC X1. https://subpac.com/x1/. Accessed 2021-03-23.

[49] Hong Tan, Robert Gray, J Jay Young, and Ryan Taylor. 2003. A haptic back display for attentional and directional cueing. (2003).

[50] Hong Z Tan, Seungmoon Choi, Frances WY Lau, and Freddy Abnousi. 2020. Methodology for Maximizing Information Transmission of Haptic Devices: A Survey. Proc. IEEE 108, 6 (2020), 945-965.

[51] H. Z. Tan, S. Choi, F. W. Y. Lau, and F. Abnousi. 2020. Methodology for Maximizing Information Transmission of Haptic Devices: A Survey. Proc. IEEE 108, 6 (2020), 945-965. https://doi.org/10.1109/JPROC.2020.2992561

[52] Yu-Chih Tung and Kang G. Shin. 2016. Expansion of Human-Phone Interface By Sensing Structure-Borne Sound Propagation. In Proceedings of the 14th Annual International Conference on Mobile Systems, Applications, and Services (Singapore, Singapore) (MobiSys '16). Association for Computing Machinery, New York, NY, USA, 277-289. https://doi.org/10.1145/2906388.2906394

[53] Jean Vroomen and Mirjam Keetels. 2010. Perception of intersensory synchrony: a tutorial review. Attention, Perception, \& Psychophysics 72, 4 (2010), 871-884.

[54] Siri Weerasooriya and Mohamad A El-Sharkawi. 1991. Identification and contro of a dc motor using back-propagation neural networks. IEEE transactions on Energy Conversion 6, 4 (1991), 663-669.

[55] Juan Wu, Jun Zhang, Ju Yan, Wei Liu, and Guangming Song. 2012. Design of a vibrotactile vest for contour perception. International fournal of Advanced Robotic Systems 9, 5 (2012), 166. 